\title{
Comparison of Forces Developed by the Leg of the Rock Lobster when Walking Free or on a Treadmill ${ }^{\star}$
}

\author{
F. Clarac $^{1}$ and H. Cruse ${ }^{2}$ \\ 1 Laboratoire de Neurobiologie comparé, Arcachon, France \\ 2 FB Biologie, Universität Kaiserslautern, Kaiserslautern, Federal Republic of Germany
}

Summary. In the free walking rock lobster the forces developed by legs 4 and 5 were investigated during the power stroke. Two orthogonal force components lying in the horizontal plane were measured. Based on these results the different tasks of the two legs during walking are discussed. The forces developed by leg 4 were compared when the animal walked freely and on a treadmill. In these two situations the results differ qualitatively as in driven walking the forces are nearly identical in a long series of consecutive steps whereas in free walking the forces can vary greatly from step to step. However, similar mean values of force were measured with those on the treadmill being somewhat higher. This shows that, although the treadmill is driven by a motor, the animal does perform active walking movements. In the treadmill situation the forces increase as the speed of treadmill motor is decreased.

\section{Introduction}

Recent investigations measure the timing of the movement of the legs in walking crustaceans. Up to now all experiments were done in evaluating films of walking animals (Clarac and Coulmance, 1971; Barnes, 1975; Sleinis and Silvey, 1980) or with electrophysiological methods (MacMillan, 1975; Ayers and Davis, 1977; Ayers and Clarac, 1978). Particularly the last one is a powerful method to obtain detailed measurements of the exact timing of the legs also in different experimental situations as e.g. after amputation of legs. However, the main meaning of the legs is to develop forces to hold and/or to move the body of the animal. Especially for animals being able to walk under water and on

* Supported by DAAD and DFG (Cr 58) for H. Cruse and by ATP (80 119.112) INSERM for F. Clarac land as some decapods do, the forces developed by the legs have to vary to a large degree and therefore can be assumed to be an important parameter for the animal when controlling the movement of the legs (Pond, 1975). Upon this parameter only qualitative results can be obtained by the methods mentioned (Burrows and Hoyle, 1973). Therefore in this paper we want to measure the forces which are developed by the leg of the rock lobster Jasus lalandii walking under water. In normal walking this animal is not using leg 1 und 2 . Also leg 3 is not always used in walking and then often in a irregular way (Chasserat and Clarac, 1980). Therefore only leg 4 and 5 are investigated here. These are also those legs which are normaly used to support the body of the resting animal. Some of the results are mentioned by Clarac (1981).

Several earlier experiments are done with animals walking on a motor driven treadmill (Ayers and Davis, 1977; Chasserat and Clarac, 1980). However, it is not clear whether in this situation the legs show active power strokes as the free walking animal does or does it merely develop weak forces and sometimes even passive movements. Therefore force measurements were made in animals walking free and walking on the treadmill in order to compare the behaviour of the animals in both situations.

\section{Methods}

Adult rock lobsters Jasus lalandii (Decapods, Crustacea) with a mean weight of $400-600 \mathrm{~g}$ (all weights are in air) were used in these experiments. The force transducer was made from two strain gauges (Philips PR 9833) which were glued at a spring steel foil $0.1 \mathrm{~mm}$ thick. The foil (15 $\mathrm{mm}$ long, $5 \mathrm{~mm}$ broad) was shown as a black bar in Fig. 1 and was fixed at an angle $\alpha$ to a plastic tube of $25 \mathrm{~mm}$ length which could be pushed over the dactylopodite and the protopodite, 


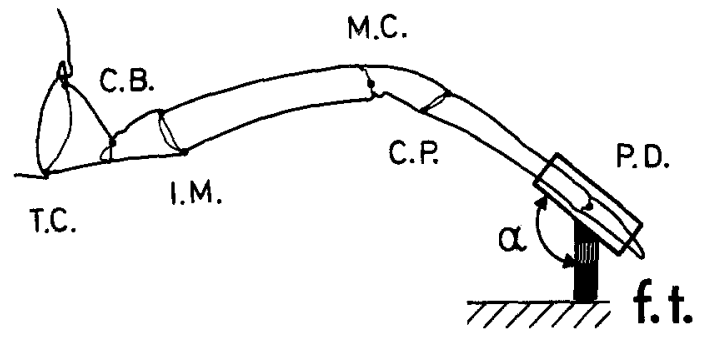

Fig. 1. The position of the force transducer I (f.t.) on leg 4 . The spring steel foil is shown as a black bar. The position of the strain gauge on the foil is shown by a small grid. T.C.: thoraco-coxal joint, C.B.: coxo-basal joint, I.M.; ischi-meropodite joint, M.C.: merocarpopodite joint, C.P.: carpo-protopodite joint, P.D.: protodactylopodite joint. For further explanation see text
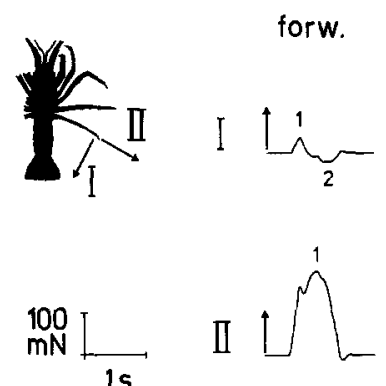

backw.
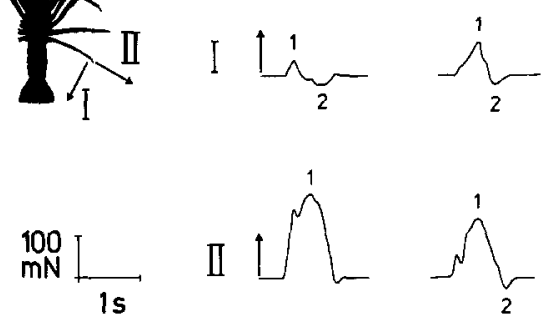

Fig. 2. Typical forces developed by leg 5 during free forward and backward walking. The inset shows the direction of the force components measured by force transducer I and II. The arrows show positive forces. It should be mentioned that this coordinate system is fixed to the leg and therefore changes direction during leg movement. The numbers label those extreme values the mean values of which are shown in Table 1

so that the $\mathrm{P}-\mathrm{D}$ joint was fixed in a straight position and the force transducer foil replaced the dactylopodite as a strut. The weight of tubing and foil detector was $40 \mathrm{mN}$. The movement of the tip of the foil was $1 \mathrm{~mm}$ when loaded with $200 \mathrm{mN}$. The wire leads were fixed to the carapace of the body and forces could be measured during the power stroke but not during return stroke of the leg.

Two independent series of experiments using two different force transducers were performed. Both force transducers were perpendicular to the walking surface during most of the power stroke. The two force transducers were used to measure different spatial components of the force. Force transducer I was positioned so that it mainly measured the forces developed by the $\mathrm{T}-\mathrm{C}$-joint $\mathrm{i}$.e. the measured force component is orientated perpendicular to the drawing plane of Fig. 1. Force transducer II was rotated about $90^{\circ}$ around the vertical axis and therefore measured the component lying in the drawing plane. The angle $\alpha$ between force transducer (long axis of the foil) and the long axis of the protopodite was about $130^{\circ}$ if not
Table 1. The forces developed by leg 5 in the free walking animal as shown in Fig. 2. Each section contains mean values, standard deviation, sample sizes $n$ and number of animals. The force values are given in $\mathrm{mN}$. The numbers refer to the extreme values labelled in Fig. 2. For further explanation see Fig. 2

\begin{tabular}{lll}
\hline Leg 5 & Forward walking & Backward walking \\
\hline Force & $1: 22( \pm 20)$ & $1: 26( \pm 31)$ \\
$\quad$ transducer I & $2:-2( \pm 10)$ & $2:-4( \pm 17)$ \\
& $n=224$ & $n=9$ \\
& 2 animals & 1 animal \\
Force & $1: 102( \pm 62)$ & $1: 119( \pm 49)$ \\
$\quad$ transducer II & $n=326$ & $2:-17( \pm 32)$ \\
& 3 animals & $n=51$ \\
& & 2 animals \\
\hline
\end{tabular}

stated otherwise. As both force transducers were positioned vertically the components lying in the horizontal plane were measured (for further explanation see Discussion).

The animals walked either freely on a horizontal surface inside a round bassin with a diameter of $2 \mathrm{~m}$ or walked on a treadmill consisting of two separate belts for right and left legs. In the latter case the animal was tethered at the carapace and the weight of the holder was counter-balanced so that the animal could choose its own distance to the ground [see Chasserat and Clarac (1980) for details]. The results were recorded on magnetic tape or directly on a pen recorder (Gould Brush 2200). In some experiments also electromyograms were recorded from the coxal promoter and remotor of the fourth leg (for details sec Clarac, 1981).

\section{Results}

Leg 5

From leg 5 recordings were obtained only in the free walking situation. Although the time course of the forces can vary greatly from step to step, one finds a typical form and some examples are shown in Fig. 2. To obtain quantitative values in a number of different steps from several animals the values of the extreme points (labelled by numbers in Fig. 2) of these force curves were measured. The mean values and the standard deviation of these force values are shown in Table 1. Concerning force transducer I there are too few steps obtained in backward walking to make the statistical values meaningful. Nevertheless one can say that in both, forward and backward walking the force values of this component are very small. Results measured by force transducer II show that in forward and backward walking the major force is directed away from the body. As leg 5 in the walking animals 
(a)

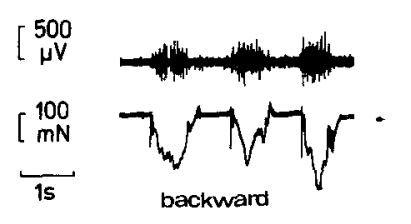

$$
\text { forw. }
$$

backw.
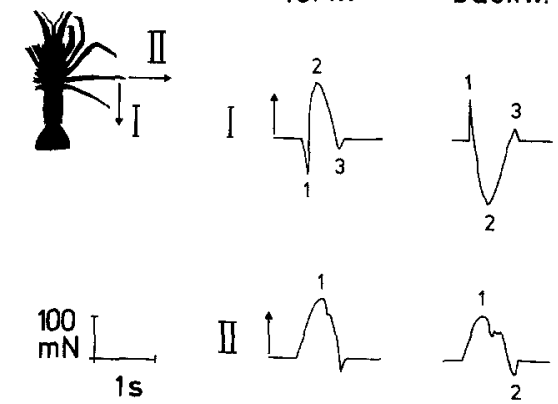

Fig. 4. Typical forces developed by leg 4 during free forward and backward walking. The numbers label those extreme values the mean values of which are shown in Table 2 . For further explanations see Fig. 2

assumes an oblique and posterior directed orientation, in forward walking these forces propel the body. In backward walking leg 5 develops forces which are opposed to the direction of movement.

\section{Leg 4}

In Fig. 3 an example of those forces measured with force transducer I are shown which were developed by leg 4 during backward (Fig. 3a) and forward (Fig. 3c) walking. In addition the electromyograms of the coxal promotor were recorded. The promotor muscle is correlated with the power stroke in backward walking and with the return stroke in forward walking. Figure 3 b shows an example where the animal first walks backward and then walks forward. Figure 4 shows typical time courses of the force of both components in the free walking animal. The quantitative values were obtained in the same way as described for leg 5 and are given in Table 2. Again the numbers refer to the extreme values labelled in Fig. 4. As can be seen in the examples of force component $I$ at the beginning of the power stroke there is often a sharp spike in the force (Fig. 4, transducer I, label 1). This is caused by the leg contacting the ground and stopping the movement of the return stroke. Label 3 (Fig. 4, transducer I) shows a small peak which corresponds to the start of the return stroke. In forward walking the main part of force is directed posteriorily and away from the body. Therefore leg 4 acts normally to move the body of the animal forward. In backward walking the forces were (c)

Fig. 3a-c. Electromyograms of the coxal promotor (upper trace) and force component I of leg 4 for a free walking animal. a Backward walking, b two steps backward then forward walking, $\mathrm{c}$ forward walking

forward

Table 2. The forces developed by leg 4 in the free walking animal as shown in Fig. 4. Each section contains mean value, standard deviation, sample sizes $n$ and number of animals. The force values are given in $\mathrm{mN}$. The numbers refer to the extreme values labelled in Fig. 4. For further explanation see Fig. 2

\begin{tabular}{lll}
\hline Leg 4 & Forward walking & Backward walking \\
\hline $\begin{array}{lll}\text { Force } \\
\text { transducer I }\end{array}$ & $1:-19( \pm 19)$ & $1: 25( \pm 34)$ \\
& $2: 86( \pm 51)$ & $2:-88( \pm 55)$ \\
& $3:-5( \pm 12)$ & $3: 9( \pm 16)$ \\
& $n=640$ & $n=172$ \\
& 6 animals & 6 animals \\
Force & $1: 102( \pm 74)$ & $1: 85( \pm 44)$ \\
transducer II & $n=302$ & $2:-15( \pm 24)$ \\
& 3 animals & $n=41$ \\
&
\end{tabular}

reversed and the force was directed anteriorly. Thus in contrast to leg 5 in backward walking leg 4 produces forces which move the body backwards.

The position of the carpopodite of leg 4 often changed during the step and from step to step and the force transducer II was not always perpendicular to the walking surface. To obtain a measure of the influence of this deviation in one animal the angle $\alpha$ between carpopodite and the force transducer (see Fig. 1) was varied between $120^{\circ}$ and $180^{\circ}$. An $\alpha$ of $\sim 120^{\circ}$ to $140^{\circ}$ corresponds to a position of the force transducer perpendicular to the walking surface. The results are shown in Table 3. Different extreme values are listed in consecutive numbers as used in Tables 1 and 2. Although in backward walking not sufficient steps were obtained, the mean values were also calculated and shown in Table 3. As expected from the geometry, the force value depends upon the angle $\alpha$. However, in the range of interest the changes are small compared to the variation from step to step. Therefore these results can be used to make qualitative statements on the walking behaviour. To show possible individual differences it should be mentioned that this animal develops less force than the three animals used for Table 2 .

In addition to free walking behaviour the same animals were fixed dorsally by the carapace and walked on a treadmill. In this situation the animals made long and regular walks and the walking speed was controlled by the experimentor. Only the activity of leg 4 was investigated. The time course of the force is 
Table 3. Means of the maximum values of the force component II of the free walking animal. $\alpha$ : angle between carpopodite and force transducer (see Fig. 1). The last row shows values obtained on the treadmill with an angle $\alpha=180^{\circ}$ and a treadmill speed of $6 \mathrm{~cm} / \mathrm{s}$. All values (given in $\mathrm{mN}$ ) were obtained from one animal

\begin{tabular}{lll}
\hline $\begin{array}{l}\text { Leg } 4 \\
\text { force transducer II }\end{array}$ & Forward walking & Backward walking \\
\hline $\begin{array}{l}\alpha=120^{\circ} \\
\text { free walking }\end{array}$ & $41( \pm 57)$ & $\begin{array}{l}1: 37( \pm 43) \\
2:-16( \pm 12) \\
n=37\end{array}$ \\
$\begin{array}{l}\alpha=140^{\circ} \\
\text { free walking }\end{array}$ & $34( \pm 42)$ & $1: 2( \pm 12)$ \\
& $n=157$ & $2:-31( \pm 18)$ \\
$\alpha=160^{\circ}$ & $25( \pm 30)$ & $n=40$ \\
free walking & $n=149$ & $1: 4( \pm 17)$ \\
& $1: 13( \pm 26)$ & $n=32$ \\
$\alpha=180^{\circ}$ & $2:-19( \pm 26)$ & $1: 1( \pm 15)$ \\
free walking & $n=258$ & $n=23$ \\
& $25( \pm 77)$ & $86( \pm 33)$ \\
$\alpha=180^{\circ}$ & $n=124$ & $n=57$ \\
treadmill & & \\
$6 \mathrm{~cm} / \mathrm{s}$ & - & \\
\hline
\end{tabular}

Table 4. The forces (component I) developed by leg 4 walking on the treadmill at different speeds and walking free. The values (given in $\mathrm{mN}$ ) were obtained from two animals

\begin{tabular}{llr}
\hline $\begin{array}{l}\text { Speed of the } \\
\text { treadmill }\end{array}$ & Forward & Backward \\
\hline $4 \mathrm{~cm} / \mathrm{s}$ & $1:-10( \pm 20)$ & $5( \pm 11)$ \\
& $2: 105( \pm 37)$ & $-158( \pm 51)$ \\
& $n=52$ & $n=20$ \\
$6 \mathrm{~cm} / \mathrm{s}$ & $1:-16( \pm 15)$ & $5( \pm 6)$ \\
& $2: 86( \pm 44)$ & $-117( \pm 65)$ \\
& $n=109$ & $n=46$ \\
$8 \mathrm{~cm} / \mathrm{s}$ & $1:-17( \pm 9)$ & $10( \pm 9)$ \\
& $2: 81( \pm 37)$ & $-137( \pm 52)$ \\
& $n=31$ & $n=22$ \\
& $1:-26( \pm 9)$ & $28( \pm 13)$ \\
$10 \mathrm{~cm} / \mathrm{s}$ & $2: 57( \pm 27)$ & $-83( \pm 62)$ \\
& $n=33$ & $n=18$ \\
& $1:-32( \pm 9)$ & $28( \pm 21)$ \\
$12 \mathrm{~cm} / \mathrm{s}$ & $2: 38( \pm 23)$ & $-58( \pm 34)$ \\
& $n=42$ & $n=16$ \\
& $1:-14( \pm 13)$ & $11( \pm 14)$ \\
Free walking & $2: 83( \pm 38)$ & $-50( \pm 38)$ \\
& $n=159$ & $n=46$ \\
\hline
\end{tabular}

independent of the speed of the treadmill but the amplitude of the force is smaller at high speeds. In Fig. 5 three examples are shown which are measured with force transducer $I$ at different speeds in forward (Fig. 5a) and backward (Fig. 5b) movement. Table 4 shows the mean values $( \pm$ S.D.) for the five different

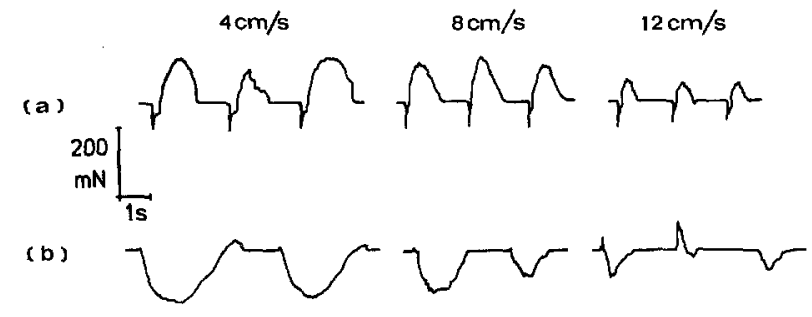

Fig. 5a and b. Force component I during forward $\mathbf{a}$ and backward $\mathbf{b}$ walks at different speeds on the treadmill

speeds investigated on the treadmill. To compare these data with those of the free walking animal immediately after the treadmill experiment the animals were again tested in the free walking situation. These mean values are also given in Table 4. Qualitatively the time course of the force is the same as in the free walking situation for forward and backward walking. The results show that the force amplitude decreases with increasing speed of the treadmill. As the speed of the free walking animal corresponds to about $6-8 \mathrm{~cm} / \mathrm{s}$ quantitative comparison will only be made within this speed range. As can be seen from Table 4 the mean values on the treadmill are somewhat larger in backward walking.

In one animal values of the force component II $\left(\alpha=180^{\circ}\right)$ obtained on the treadmill were compared with those of the free walking situation (Table 3). Again larger values were found in backward walking.

In another experiment both fourth legs on the left (L4) and on the right side (R4) of the body carried force transducers of type I. As expected from the first experiments right and left legs in free walking animals could develop very different force values so that during a series of several consecutive steps one side could show large forces and the other small or even zero values. This result was also found when the animals walked on the treadmill. In this situation it is even more obvious as such an asymmetry can persist for a long series of steps, so that either the right side or the left side of the body develops stronger forces. On the treadmill this behaviour can be influenced experimentally in the following way. When the two belts for left and right legs are driven with different speed the legs on the slower belt develop large forces while those on the faster side show small sometimes even negative forces. By inverting the speed difference this behaviour is also inverted. This does not always happen immediately but after $5-10$ steps the animal shows larger forces on the slower belt.

It should be mentioned that on the treadmill with the same speed on both sides sometimes force time courses were found which differ from those described here. Particularly at the beginning of the experiment the legs sometimes tried to oppose the movement of the treadmill either on one or both sides of the body. 
This behaviour was easy to detect as it occurred regularly over a series of several steps and then within one or two steps changed to the kind of behaviour investigated here.

\section{Discussion}

The results are concerned with two questions. One is the comparison between the different functions of leg 4 and leg 5 during free forward and backward walking. The other is the comparison between free walking and driven walking of leg 4 . However, at first the method applied here should be discussed.

\subsection{Methods}

In the method used here the P-D joint of the leg under examination could not be moved by the animal. This may influence the behaviour of the measured leg. In Cardisoma guanhumi for example the $\mathrm{P}-\mathrm{D}$ joint plays an important role in lateral walking (Barnes et al., 1972). However, lateral walking was not examined here and observation of the animal did not show any differences in the behaviour of the measured leg compared with that of the other legs. This is supported by the fact that the angular variation of the $\mathrm{P}-\mathrm{D}$ joint is of minor importance in forward and backward walking. Most important in this situation are the $\mathrm{T}-\mathrm{C}$ and $\mathrm{C}-\mathrm{B}$ joints while the $\mathrm{C}-\mathrm{B}, \mathrm{M}-\mathrm{C}$ and accessorily $\mathrm{P}-\mathrm{D}$ joints are active in lateral walking (Ayers and Davis, 1977; Ayers and Clarac, 1980). With the present method the force components are measured in a coordinate system fixed relative to the two distal segments of the leg, the propodite and dactylopodite. This means that the forces can be assigned to the muscle systems of different joints. The force component I describes forces developed primarily by the muscles of the $\mathrm{T}-\mathrm{C}$ joint (see Fig. 1) as it measures the force component which is orthogonal to the axis of rotation of this joint during the normal walking movements. The force component II corresponds to the forces developed by the $\mathrm{M}-\mathrm{C}$ joint only when $\alpha=180^{\circ}$. In the other positions both, the $\mathrm{M}-\mathrm{C}$ joint and the $\mathrm{C}-\mathrm{B}$ joint contribute to the measured forces. The two parts cannot be separated when only one force component is measured. The axis of rotation of the other joints are arranged obliquely and therefore contribute to both force components. However, force values can only assigned qualitatively as the length of the lever arm was not measured here.

As the force transducers are fixed to the leg they do not have the same spatial orientation during the whole power stroke. This means that the force components measured do not always lie in the horizontal plane. Experiments with different positions of the force transducer showed that the differences of the force values are small compared to the variation which occurs from step to step. Therefore the force values measured here can be regarded as a first approximation to those force components which lie in the horizontal plane and accelerate or decelerate the body in this plane.

\subsection{The Function of the Legs}

The results show that in leg 5 the main forces during the power stroke are developed in the $\mathrm{C}-\mathrm{B}$ joint and/or the $\mathrm{M}-\mathrm{C}$ joint (Table 1, II : forw. $102 \mathrm{mN}$, backw. $119 \mathrm{mN}$ ) whereas the $\mathrm{T}-\mathrm{C}$ joint is of minor importance (Table 1, I: forw. $22 \mathrm{mN}$, backw. $-26 \mathrm{mN}$ ). In leg 4 the $\mathrm{T}-\mathrm{C}$ joint (Table 2, I : forw. $86 \mathrm{mN}$, backw. $-88 \mathrm{mN}$ ) as well as the $\mathrm{C}-\mathrm{B}$ joint and/or the $\mathrm{M}-\mathrm{C}$ joint (Table 2, II : forw. $102 \mathrm{mN}$, backw. $85 \mathrm{mN}$ ) produce considerable forces during the power stroke. The measurements of force component II with variing angle $\alpha$ suggests that the $\mathrm{M}-\mathrm{C}$ joint provides only a smaller amount of the whole force (Table $3, \alpha=180^{\circ}$ : forw. $13 \mathrm{mN},-19 \mathrm{mN}$, backw. $-33 \mathrm{mN}$ ). However, the latter measurements were obtained from only one animal.

What do the individual legs contribute to the movement of the whole animal? Leg 4 accelerates the body in the anterior direction during forward walking and in the posterior direction during backward walking. In addition in both situations a sideways force is produced. Together with the corresponding force component of the legs of the other side of the body a threecentered arch is formed which is capable of supporting the body (Cruse, 1976). During forward walking leg 5 develops a force which accelerates the body in the anterior direction. However, even during backward walking the leg develops similar forces in the anterior direction although the $\mathrm{M}-\mathrm{C}$ joint is bent during the power stroke. This means that leg 5 in backward walking is mainly used as a strut against which the body is pushed by leg 4 and possibly by leg 3 and suggests that leg 4 is of greater importance in controlling the direction of movement. The use of legs as struts was first suggested by Hughes (1952) and this has also been experimentally verified in stick insects when walking on a horizontal plane (Cruse, 1976). In addition this result shows that one cannot conclude from the movement of the joint the forces that are being developed in this joint. This is only possible when the forces are measured directly for, as stated in the introduction also from electrophysiological recordings no quantitative information can be obtained concerning the amount of forces developed in this joint.

\subsection{Comparison Between Free and Treadmill Walking}

Although on the motor driven treadmill the legs might be passively moved during the stance phase the results show that in this situation the animals do actively push 
against the belt in the power stroke direction. The time course of the forces and their amplitudes are not different to those of free walking animals. This supports the results of Ayers and Davis (1977) obtained with electrophysiological methods and shows clearly that animals on a treadmill show real active walking behaviour. One difference between free walking and driven walking seems to be that for low speeds of the treadmill the forces are higher than in the free walking situation. Results of Ayers and Davis (1977) seem to show a corresponding tendency which is however much less obvious when using electrophysiological techniques. The increase of the force might be the result of a conflict between the walking speed intended by the animal and the actual speed of the treadmill (Chasserat and Clarac, 1980; Cruse and Clarac, in preparation). One possible explanation of this observation is to assume a servomechanism controlling the position of each leg. Such a system would increase the force if the speed of the treadmill is less than the intended walking speed.

Another difference seems to be that in free walking the force values show a considerable variation from step to step whereas in walks on the treadmill the time courses are much more stereotyped. The reason might be that the treadmill constrains the animal to walk more continuously and in only one direction. In contrast a free walking animal can stop, turn or walk lateral. Therefore the forces are expected to show a large variation. Also in EMG recordings a greater variability is found (Clarac, in preparation). However, even on the treadmill sometimes the power stroke can be partly or totally passive (Clarac and Chasserat, in preparation). Even in such cases on the treadmill the behaviour is more stable over a series of steps whereas in free walking strong variations can occur from step to step.

On the treadmill as in the free walking situation differences in the forces developed by right and left legs can occur but these asymmetries are more regular and can be manipulated experimentally. In both situations this asymmetry between right and left legs probably corresponds to the animal tending to turn or walk sideways.

\section{References}

Ayers, J.C., Clarac, F.: Neuromuscular strategies underlying different behavioural acts in a multifunctional crustacean leg joint. J. Comp. Physiol. 128, 81-94 (1978)

Ayers, J.C., Davis, W.J.: Neuronal control of locomotion in the lobster Homarus americanus. I. Motor programs for forward and backward walking. J. Comp. Physiol. 115, 1-27 (1977)

Barnes, W.J.P.: Leg coordination during walking in the crab, Uca pugnax. J. Comp. Physiol. 96, 237-256 (1975)

Barnes, W.J.P., Spirito, C.P., Evoy, W.H. : Neural control of walking in the crab, Cardiosoma guanhumi. II. Role of resistance reflexes in walking. Z. Vergl. Physiol. 76, 16-31 (1972)

Burrows, M., Hoyle, G.: The mechanism of rapid running in the ghost crab Ocypode ceratophtalma. J. Exp. Biol. 58, 327-349 (1973)

Chasserat, C., Clarac, F.: Interlimb coordinating factors during driven walking in Crustacea. J. Comp. Physiol. 139, 293-306 (1980)

Clarac, F.: Decapod crustacean leg coordination during walking. In: Locomotion and energetics in arthropods. Herreid, C.F., Fourtner, C.R. (eds.). New York, London: Plenum Press 1981

Clarac, F., Coulmance, M. : La marche laterale du crabe (Carcinus). Z. Vergl. Physiol. 73, 408-438 (1971)

Cruse, $H$.: The function of the legs in the free walking stick insect, Carausitus morosus. J. Comp. Physiol. 112, 235-262 (1976)

Clarac, F., Chasserat, C.: Right and left coordination in a rock lobster during driven walking on two independent belts (in preparation)

Cruse, H., Clarac, F.: The control of walking movements in the leg of the rock lobster (in preparation)

Hughes, G.M.: The coordination of insect movements. I. The walking movements of insects. J. Exp. Biol. 29, 267-285 (1952)

MacMillan, D.L.: A physiological analysis of walking in the American lobster, Homarus americanus. Phil. Trans. R. Soc. B270, 1-59 (1975)

Pond, C.M.: The role of the walking legs in aquatic and terrestrial locomotion in the cray fish Austropotamobius pallipes. J. Exp. Biol. 62, 447-454 (1975)

Sleinis, S., Silvey, G.E.: Locomotion in forward walking crab. J. Comp. Physiol. 136, 301-312 (1980)

Received: July 13, 1981

Dr. F. Clarac

Laboratoire de Neurobiologie comparée

2, Rue du Prof. Jolyet

F-33120 Arachon

France

Prof. Dr. H. Cruse

Fakultät Biologie der Universität

Postfach 8640

D-4800 Bielefeld 1

Federal Republic of Germany 